\title{
A MASSIVE WHITE DWARF COMPANION TO THE ECCENTRIC BINARY PULSAR SYSTEM PSR B2303+46
}

\author{
M. H. VAN KERKWIJK \\ Astronomical Institute, Utrecht University, P. O. Box 80000, 3508 TA Utrecht, The Netherlands \\ AND \\ S. R. KULKARNI \\ Palomar Observatory 105-24, California Institute of Technology, Pasadena, CA 91125 \\ Received 1999 January 11; accepted 1999 February 22; published 1999 March 9
}

\begin{abstract}
Pulsars in close, eccentric binary systems are usually assumed to have another neutron star as a companion. These double neutron star binaries have proven to be the best laboratories for experimental general relativity and are the most secure candidates for gravitational wave interferometers. We present deep $B, V$, and $R$ images of the field containing the eccentric binary pulsar system PSR B2303+46. We find a faint, blue object $[B=$ $\left.26.60 \pm 0.09 ;(B-R)_{0}=-0.4 \pm 0.2\right]$ coincident with the timing position. We suggest that this object is the optical counterpart to the PSR B2303+46 system. The counterpart is too bright to reflect emission from the pulsar or a neutron star companion. Most likely, the companion of PSR B2303+46 is not a neutron star but a massive white dwarf. We show that the observations are consistent with a hot white dwarf companion $\left(T_{\text {eff }} \geq 5 \times 10^{4} \mathrm{~K}\right)$ with cooling age equal the characteristic age of the pulsar $\left(t_{\text {cool }} \simeq 30 \mathrm{Myr}\right)$ and mass within the range set by timing observations and the Chandrasekhar mass $\left(1.2<M_{\mathrm{C}}<1.4 M_{\odot}\right)$. Given the eccentric orbit, the white dwarf must have formed before the neutron star, from what was originally the more massive star in the binary. Due to mass transfer, the originally less massive star could become sufficiently massive to end its life in a supernova explosion and form the radio pulsar. We constrain the mass of the pulsar to be in the range $1.24<M_{\mathrm{PSR}}<1.44 M_{\odot}$.
\end{abstract}

Subject headings: binaries: close — pulsars: individual (PSR B2303+46) — stars: evolution

\section{INTRODUCTION}

Binaries in which a radio pulsar is in a close, eccentric orbit around a compact companion have provided the best astronomical tests of general relativity and the most accurate neutron star mass determinations (Taylor et al. 1992), are the most secure targets for gravitational wave interferometers such as the Laser Interferometer Gravitational-wave Observatory (Abramovici et al. 1992), and may be the progenitors of the enigmatic gamma-ray bursts (Piran 1997). Especially for the latter two topics, the nature of the compact companion is of vital importance. Fortunately, optical observations can distinguish the three possibilities: white dwarfs can be detected out to large distances, whereas neutron stars are rather dim and black holes are not detectable at all.

It has become general practice to identify as a double neutron star system any high-eccentricity binary pulsar for which the inferred companion mass is $\sim 1.4 M_{\odot}$ (for a review, see van den Heuvel 1995). This is based on the evolutionary scenario for these systems. Briefly, it starts with an early-type binary. The primary (the more massive star) evolves first, transfers some mass to the secondary, explodes as a supernova, and forms a neutron star. Next, the secondary evolves and starts to transfer matter to the neutron star. The accretion causes "recycling:" spin-up and-in a way not well understood-a reduction in magnetic field strength. The mass transfer will be unstable, leading to a common-envelope phase. The orbit will necessarily be circularized; it can only become eccentric if the secondary is sufficiently massive to explode and form a neutron star in turn. (Otherwise, a white dwarf is left in a circular orbit, as observed for other binary pulsars.) Thus, if the observed radio pulsar can be shown to be the first-formed, recycled neutron star, the companion must be a neutron star as well.

In four of the six presumed double neutron star systems-PSR B1913+16, PSR J1518+4904, PSR B1534+12, and PSR B2127+11C - the pulsars indeed appear recycled: the spin periods are shorter and the inferred magnetic fields are weaker than for ordinary pulsars (tens of milliseconds vs. $\sim 1 \mathrm{~s}$, and $\sim 10^{10} \mathrm{G}$ vs. $\gtrsim 10^{12} \mathrm{G}$ ). However, the pulsars in the other two systems, PSR B1820-11 and PSR B2303+46, show no clear sign of recycling. For the former, Phinney \& Verbunt (1991) suggested that the companion was not a neutron star but a low-mass main-sequence star and that the system will eventually become a low-mass X-ray binary. The latter, PSR B2303+46, is the subject of this Letter.

\section{PSR B2303+46}

PSR B2303+46 is in a 12.3 day, highly eccentric $(e=$ 0.66) orbit (Stokes, Taylor, \& Dewey 1985). Periastron advance is observed, from which one infers a total mass of the system $M_{\mathrm{PSR}}+M_{\mathrm{C}}=2.64 \pm 0.05 M_{\odot}$ as well as, in combination with the mass function, the limits $M_{\mathrm{PSR}}<1.44 M_{\odot}$ and $M_{C}>$ $1.20 M_{\odot}$ (Thorsett et al. 1993; Thorsett \& Chakrabarty 1999).

The pulsar period $(P=1.06 \mathrm{~s})$ and inferred magnetic field strength $\left(B \simeq 8 \times 10^{11} \mathrm{G}\right)$ allow the possibility of mild recycling. Given the small characteristic age, $\tau_{\mathrm{PSR}}=$ $P / 2 \dot{P} \simeq 30 \mathrm{Myr}$, radio pulsations of the presumed neutron star companion have been searched for, but with no success. Kulkarni (1988) carried out optical observations with the Palomar 200 inch $(5 \mathrm{~m})$ telescope, but found no counterpart down to $R=26 \mathrm{mag}$; this was seen as confirmation of the scenario outlined above.

The pulsar parameters, however, are also consistent with those of ordinary pulsars. Thus, the pulsar could have formed after the companion completed its evolution. If so, it may be the only neutron star in the binary, the companion being a white dwarf. This requires a twist to the evolutionary scenario, in which one starts with two stars with masses (slightly) below the critical mass $M_{\text {crit }}\left(\sim 8 M_{\odot}\right.$; Koester \& Reimers 1996) re- 


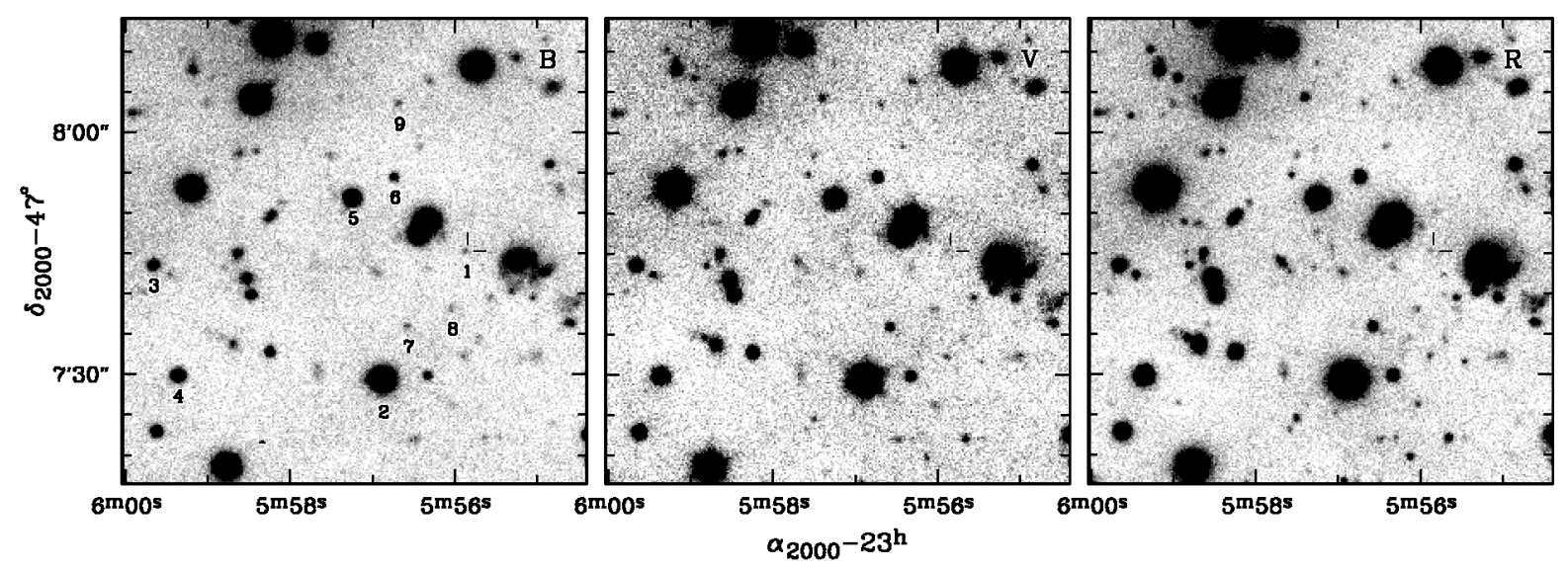

FIG. 1.-Stacked $B, V$, and $R$ images of the localization of PSR B2303+46. In each panel, the timing position is indicated with tick marks (of size 1".5). Stars mentioned in the text are labeled below their image.

quired to evolve to a neutron star. In due course, the primary evolves, transfers matter to the secondary, and forms a white dwarf. Now, if the mass transfer increased the secondary mass beyond $M_{\text {crit }}$, it can explode and form a neutron star, resulting in a binary with an older white dwarf and a younger neutron star in a highly eccentric orbit.

We were reminded of this possibility by R. A. M. J. Wijers (1997, private communication), who wondered whether it could be verified observationally. At a distance of $4.3 \mathrm{kpc}$ (inferred from the pulsar dispersion measure) and for a cooling age of $30 \operatorname{Myr}\left(\tau_{\text {PSR }}\right)$, a $1.2 M_{\odot}$ white dwarf counterpart would have $V \simeq 25$. This is excluded by the limit mentioned above, but, perhaps fortuitously, we had forgotten about this result. Here, we report new, deeper optical observations with the Keck telescope, which show a possible counterpart to the PSR B2303+46 system.

\section{OPTICAL OBSERVATIONS}

We imaged the field containing PSR B2303+46 with the Low-Resolution Imaging Spectrograph (Oke et al. 1995) at the Keck II telescope, on the nights of 1997 November 28 and 29 (UT). On November 28, three $600 \mathrm{~s}$ exposures were obtained

TABLE 1

Photometry AND Astrometry

\begin{tabular}{|c|c|c|c|c|c|}
\hline Star & $\alpha_{\mathrm{J} 2000}$ & $\delta_{\mathrm{J} 2000}$ & $\begin{array}{c}B \\
(\mathrm{mag})\end{array}$ & $\begin{array}{c}V \\
\text { (mag) }\end{array}$ & $\begin{array}{c}R \\
(\mathrm{mag})\end{array}$ \\
\hline $\mathrm{PSR}^{\mathrm{a}}$ & 230555.842 & 470745.32 & & & \\
\hline $1 \ldots$ & 230555.869 & 470745.30 & $26.60(9)$ & 26.91(20) & $26.65(16)$ \\
\hline 2 & 230556.875 & 470729.37 & $19.84(2)$ & $18.89(2)$ & $\cdots$ \\
\hline 3 & 230559.665 & 470743.54 & $24.08(2)$ & $22.64(2)$ & $21.72(2)$ \\
\hline 4 & 230559.362 & 470729.88 & $22.79(2)$ & $21.16(2)$ & $20.10(2)$ \\
\hline 5 & 230557.242 & 470751.89 & $22.00(2)$ & $20.62(2)$ & $19.69(2)$ \\
\hline 6 & 230556.734 & 470754.47 & $25.18(3)$ & $23.46(2)$ & $22.21(2)$ \\
\hline 7 & 230556.574 & 470735.96 & $26.51(8)$ & $24.74(3)$ & $23.40(2)$ \\
\hline 8 & 230556.036 & 470738.18 & 26.73(10) & $26.39(11)$ & $25.99(9)$ \\
\hline $9 \ldots \ldots \ldots$ & 230556.682 & 470803.55 & $26.43(8)$ & $26.20(9)$ & $25.87(8)$ \\
\hline
\end{tabular}

Note. - Star 1 is the proposed optical counterpart; other stars are discussed in the text. The measurement uncertainties for the positions of optical objects are $\lesssim 0.004$ and $\leqq 0$ ".04. Possible uncertainties in the tie to astrometric systems are discussed in $\S 3$. The uncertainties in the photometry are indicated by the numbers in brackets. Star 2 has no $R$-band magnitude, since it was overexposed in the $R$-band images. Units of right ascension are hours, minutes, and seconds, and units of declination are degrees, arcminutes, and arcseconds.

This line gives the timing position for PSR B2303+46 (Thorsett et al. 1993); the uncertainties are 0.017 and $0^{\prime \prime} .17$. in the $R$ band, and two $900 \mathrm{~s}$ exposures in $B$. On November 29 , one $450 \mathrm{~s}$ and four $600 \mathrm{~s}$ in $R$, three $900 \mathrm{~s}$ in $B$, and five $600 \mathrm{~s}$ exposures in $V$ were taken. All images were taken at air mass less than 1.4. The skies were clear on the second night, but the first night was plagued by cirrus.

The reduction was done as described by Kulkarni \& van Kerkwijk (1998) for the field of RX J0720.4-3125, which was observed on the same nights. For the photometric calibration, we used Landolt fields: in $B$ and $R$, the four listed in Table 1 of Kulkarni \& van Kerkwijk; in $V$, the first two only. We estimate uncertainties of $\lesssim 0.02 \mathrm{mag}$ in the zero points.

For the astrometry, we selected from the USNO-A2.0 catalog (Monet et al. 1998) all 163 stars that overlapped with a $10 \mathrm{~s}$ $R$-band image. We measured their centroids and corrected for instrumental distortion using a bi-cubic function determined by J. Cohen (1997, private communication). With the plate scale known accurately, we fitted only for the zero points in each coordinate and the position angle on the sky. After rejecting seven outliers (residual larger than 0 ."8), the rms residuals were 0 ".20 in each coordinate. The astrometry was transferred to the stacked $B, V$, and $R$ images using 28 transfer stars close to the pulsar position, solving again for rotation and zero points. The rms residuals were $\leqslant 0$ ".04.

Close to the timing position of PSR B2303+46, we found one faint, relatively blue object, hereafter star 1 (see Fig. 1 and Table 1). To verify whether the respective positions are consistent with each other, one has to take into account the measurement uncertainties $\left(\sigma_{1}=0.04, \sigma_{\mathrm{PSR}}=0\right.$ ". 17 in each coordinate) as well as the extent to which the two positions are on the same astrometric system. The USNO-A2.0 catalog is tied to the International Celestial Reference System (ICRS) as realized by the Tycho-based ACT catalog (see Monet et al. 1998), while the DE200 dynamical ephemeris-on which the pulsar timing position is based-is close to the ICRS as well (Folkner et al. 1994). We expect the frame difference between DE200 and USNO-A2.0 to be considerably less than $\sigma_{\mathrm{PSR}}$. Star 1 is offset by 0 .'28 from the timing position, i.e., well within the $95 \%$ confidence radius of 0.4 that one infers from $\sigma_{\mathrm{PSR}}$ alone.

For the photometry, we used a simple point-spread function fitting method, which takes into account gradients in the sky level (see Kulkarni \& van Kerkwijk 1998). First, we used aperture photometry on images from November 29 to measure the instrumental magnitudes for relatively isolated, brighter "secondary" stars (stars 2-6 in $B, V$; stars 3-6 in $R$, because 
star 2 was overexposed; Fig. 1 and Table 1). Next, we extracted $21 \times 21$ pixel $\left(44^{\prime \prime} 5 \times 4\right.$ ".5) regions from the stacked images around stars $2-6$, the candidate (star 1), and three other faint objects (stars 7-9). We fitted these to a two-dimensional Gaussian distribution on top of a plane with an arbitrary tilt and determined the average FWHM for the secondary stars. We then refitted all objects, keeping the FWHM fixed at the average, and used the amplitudes of the Gaussian distributions to determine relative magnitudes. Finally, the difference with the aperture results for the secondary stars was used to calculate instrumental magnitudes for stars $1,7,8$, and 9, and all magnitudes were calibrated using the solution found from the Landolt stars. The results are listed in Table 1 . In order to verify our procedures, we also determined $B$ and $R$ magnitudes for stars $1,7,8$, and 9 from the stacked images from November 28 and 29 separately; these gave consistent results.

\section{A MASSIVE WHITE DWARF COMPANION}

The probability that star 1 is a background object that happens to be within the $0.5 \operatorname{arcsec}^{2}$ error region (95\% confidence) of the timing position of PSR B2303+46 is about 5\%, i.e., not particularly low. With $B-R=-0.05 \pm 0.18$, however, star 1 is bluer than all other faint stars in the field. In our images, the bluest other objects have $B-R \simeq 0.6$ (e.g., stars 8 and 9). Of these, there are only a few per square arcminute, and the chance coincidence probability is less than $0.1 \%$. The probability for an object as blue as star 1 is lower still, and, therefore, we believe star 1 is the optical counterpart to PSR B2303+46.

It is unlikely that the optical emission is caused by the pulsar or by a neutron star companion. Thermal emission from a neutron star could reproduce the colors, but it would be much too faint: the known sources have similar magnitudes, but are all nearby (for a recent compilation, see Mignani 1998). Nonthermal emission can lead to brighter sources, but only for young pulsars and generally with colors that are too red. This leads us to propose that the companion of PSR B2303+46 is a massive white dwarf and that star 1 is its optical counterpart.

In order to verify whether our observations are consistent with a white dwarf companion, we need to estimate the expected brightness. This is possible using cooling models for white dwarfs, provided we have estimates for the white dwarf mass, composition, and age, as well as for the distance and reddening. We will discuss these in turn.

The mass of the white dwarf companion has a strict lower bound of $1.2 M_{\odot}$ (inferred from timing; $\S 2$ ). An equally strict upper bound of $1.4 M_{\odot}$ is set by the Chandrasekhar mass.

The age of the white dwarf is the sum of $t_{\mathrm{SN}}$, the time that elapsed between the formation of the white dwarf and the supernova explosion, and $t_{\mathrm{PSR}}$, the age of the pulsar (see $\S 2$ ). An upper limit to $t_{\mathrm{SN}}$ is set by the total lifetime of an $8 M_{\odot}\left(M_{\text {crit }}\right)$ star, i.e., $t_{\mathrm{SN}}<40 \mathrm{Myr}$ (e.g., Schaller et al. 1992). An upper limit to $t_{\mathrm{PSR}}$ is set by the characteristic age (but see $\S 5$ ), i.e., $t_{\mathrm{PSR}} \lesssim \tau_{\mathrm{PSR}}=30 \mathrm{Myr}$.

The cooling age of the white dwarf may equal its actual age, or $t_{\mathrm{cool}}=t_{\mathrm{SN}}+t_{\mathrm{PSR}}<70 \mathrm{Myr}$. It is quite likely, however, that the white dwarf was reheated when, prior to the supernova explosion, the system went through a common-envelope phase (required to account for the current small orbital size). If so, $t_{\mathrm{SN}}$ is irrelevant, and $t_{\text {cool }} \simeq t_{\mathrm{PSR}} \lesssim 30 \mathrm{Myr}$.

The composition of massive white dwarfs is still uncertain, but observations of novae indicate that both $\mathrm{C}+\mathrm{O}$ and
$\mathrm{O}+\mathrm{Ne}+\mathrm{Mg}$ are possible (Starrfield 1989). ${ }^{1}$ Fortunately, the mass-radius relations are very similar (as inferred from Hamada \& Salpeter 1961), and hence the cooling tracks should be very similar as well. For massive white dwarfs, effects related to the composition of the atmosphere are expected to be small as well (Wood 1995). For completeness, we note that a hydrogen atmosphere seems likely, since some hydrogen will have been accreted during the common-envelope phase.

The distance toward PSR B2303 +46 can be constrained from the observed dispersion measure (DM) of $62 \mathrm{~cm}^{-3} \mathrm{pc}$. Using the Galactic model for the distribution of free electrons of Taylor \& Cordes (1993), we find that in the pulsar's direction $\left(l^{I I}=105^{\circ} .41, b^{I I}=-11^{\circ} .93\right)$, the predicted DM is consistent with the observed one for any distance $d>2.5 \mathrm{kpc}$. There is no upper limit on the distance, since the predicted maximum DM (for objects well outside the electron layer) is $78 \pm 20$ $\mathrm{cm}^{-3}$ pc. At the lower limit, the predicted DM is $41 \pm 10$ $\mathrm{cm}^{-3} \mathrm{pc}$.

The reddening along the line of sight, estimated from dust infrared emission, is $E_{B-V}=0.22 \pm 0.03$ (Schlegel, Finkbeiner, \& Davis 1998). One infers $A_{B}=0.95 \pm 0.13, A_{V}=$ $0.73 \pm 0.10$, and $A_{R}=0.59 \pm 0.08$. For any likely identification, star 1 is well out of the Galactic plane, and the full reddening should be taken into account.

With the above, we are in a position to estimate the cooling flux from a presumed white dwarf companion to PSR B2303+46. From the cooling tracks of Benvenuto \& Althaus (1999), we find that after $30 \mathrm{Myr}$ a $1.2 M_{\odot} \mathrm{C}+\mathrm{O}$ white dwarf has cooled down to $M_{\text {bol }}=6.5$ and $T_{\text {eff }}=5 \times 10^{4} \mathrm{~K}$. (Similar results are obtained extrapolating tracks of Wood 1995). From the atmospheric models of Bergeron, Wesemael, \& Beauchamp (1995), we find for this temperature $B C_{V}=-4.5$ and Rayleigh-Jeans-like colors $(B-V)_{0}=-0.28,(V-R)_{0}=$ -0.14 (for a hydrogen atmosphere; differences for other atmospheric compositions should be small). Thus, one expects $M_{B}=10.7$ and $(B-R)_{0}=-0.42$.

For a cooling age of $70 \mathrm{Myr}$, we find $M_{\mathrm{bol}}=7.5, T_{\mathrm{eff}}=$ $4 \times 10^{4} \mathrm{~K}, M_{B}=11.0$, and $(B-R)_{0}=-0.42$. For higher masses, predictions are harder to make, since no cooling tracks are available. Extrapolating, we expect more massive white dwarfs to have smaller radii, but to be hotter at the same age. The likely net effect will be that they are brighter bolometrically, but fainter in the optical (showing the same color). A conservative lower limit, $M_{B}<13.4$, is inferred by taking into account the change in radius only, from $\sim 0.006 R_{\odot}$ for a $1.2 M_{\odot}$ white dwarf to $\sim 0.002 R_{\odot}$ for one at the Chandrasekhar mass (Hamada \& Salpeter 1961).

In summary, we expect that a white dwarf companion will have $(B-R)_{0}=-0.42$ and $13.4>M_{B} \geqslant 10.7$. It can be brighter only if $t_{\text {cool }}$ is substantially smaller than $\tau_{\mathrm{PSR}}$, which we consider unlikely. The expected reddened color, $B-R=-0.06$ \pm 0.05 , is consistent with the observations. The expected distance modulus is $12.3<B-M_{B}-A_{B} \lesssim 15$, corresponding to a distance of $3<d \lesssim 10 \mathrm{kpc}$, which is consistent with the lower limit set by the dispersion measure.

\section{RAMIFICATIONS}

We presented Keck imaging of PSR B2303+46, in which we identified a faint blue object, star 1 , coincident with the

\footnotetext{
${ }^{1}$ For some massive white dwarfs, masses and radii indicate Fe composition (Provencal et al. 1998). This is excluded here: for Fe white dwarfs, $M_{\max }=$ $1.1 M_{\odot}$ (Hamada \& Salpeter 1961).
} 
precise timing position. We have shown that the companion to PSR B2303 + 46 could be a hot, massive white dwarf, with star 1 its optical counterpart. We have outlined how the evolution of a binary composed of two stars with masses close to but below $M_{\text {crit }}$ could lead to the formation of a system like PSR B2303+46.

The system may help calibrate different chronometers. With a temperature measurement from UV observations (Hubble Space Telescope time granted), cooling models will provide a lower limit to $t_{\text {cool }}$. This may allow a test of pulsar braking: if one finds $t_{\text {cool }} \gg t_{\mathrm{SN}}+\tau_{\mathrm{PSR}}$, the braking index $n$ has to be the culprit. The braking index enters via $\tau_{\mathrm{PSR}}=P /(n-1) \dot{P}$. Usually, $n=3$ is assumed (as we have done above), which is valid for a dipole rotating in vacuo. However, this has not been verified observationally. Indeed, all measurements give $n<3$, although these were for some young, fast pulsars, for which $n<3$ is perhaps expected (Melatos 1997). It would be interesting as well to find $t_{\text {cool }} \ll \tau_{\text {PSR }}$ : this would imply that pulsars do not have to be born with short spin periods.

If the companion is indeed a white dwarf, both the lower and the upper limit to its mass are interesting. The upper limit, in combination with the total mass, corresponds to a lower limit to the mass of the pulsar. Combined with the upper limit set by timing $(\S 2)$, one finds $1.24<M_{\mathrm{PSR}}<1.44 M_{\odot}$.

The lower limit of $1.2 M_{\odot}$ makes the companion interesting as a white dwarf. Masses greater than $1 M_{\odot}$ have also been inferred for about a dozen field white dwarfs, many discovered only recently from extreme UV sky surveys (Marsh et al. 1997; Vennes et al. 1997; Finley, Koester, \& Basri 1997). It is not clear, however, whether these have been formed from massive stars, for the following reasons. (1) Quite a few, especially the more massive ones, have strong magnetic fields, which is unusual; also, it makes the mass estimates, which are based on line broadening, more uncertain (e.g., Schmidt et al. 1992;
Ferrario, Vennes, \& Wickramasinghe 1998). (2) Most are hot and therefore young; combined with the short lifetimes of stars massive enough to form them, one would expect to find them in young star clusters, not in the field (Bergeron et al. 1991). (3) In at least one object, GD 50, unexpected traces of helium in the spectrum as well as evidence for a high rotation rate have been found (Vennes, Bowyer, \& Dupuis 1996). (4) The massive white dwarfs appear to form not just a tail of the distribution of white dwarf masses, but rather a separate peak (Finley et al. 1997). These reasons have led to the speculation that at least some of these field massive white dwarfs are not the product of single-star evolution, but rather the result of mergers of two ordinary $0.6 M_{\odot}$ white dwarfs (references cited above).

White dwarfs in young star clusters and binaries almost certainly are the product of massive stars, but these have masses up to $\sim 1 M_{\odot}$ only (in NGC 2516: Koester \& Reimers 1996; in Sirius: Gatewood \& Gatewood 1978; Provencal et al. 1998). In contrast, the massive white dwarf companion of PSR B2303 + 46 has almost certainly descended from a massive star, and it is undeniably massive - a statement that can be made given the exquisite precision of pulsar timing.

We are grateful to Dave Monet for providing us with preliminary astrometric results from USNO-A2.0. We acknowledge support by a fellowship of the Royal Netherlands Academy of Arts and Sciences (M. H. v. K.) and by grants from NASA and NSF (S. R. K.). The observations reported here were obtained at the W. M. Keck Observatory, which is operated by the California Association for Research in Astronomy, a scientific partnership among California Institute of Technology, the University of California, and the NASA. It was made possible by the generous financial support of the W. M. Keck Foundation.

\section{REFERENCES}

Abramovici, A., et al. 1992, Science, 256, 325

Benvenuto, O. G., \& Althaus, L. G. 1999, MNRAS, 303, 30

Bergeron, P., Kidder, K. M., Holberg, J. B., Liebert, J., Wesemael, F., \& Saffer, R. A. 1991, ApJ, 372, 267

Bergeron, P., Wesemael, F., \& Beauchamp, A. 1995, PASP, 107, 1047

Ferrario, L., Vennes, S., \& Wickramasinghe, D. T. 1998, MNRAS, 299, L1

Finley, D. S., Koester, D., \& Basri, G. 1997, ApJ, 488, 375

Folkner, W. M., Charlot, P., Finger, M. H., Williams, J. G., Sovers, O. J., Newhall, X., \& Standish, E. M., Jr. 1994, A\&A, 287, 279

Gatewood, G. D., \& Gatewood, C. V. 1978, ApJ, 225, 191

Hamada, T., \& Salpeter, E. E. 1961, ApJ, 134, 683

Koester, D., \& Reimers, D. 1996, A\&A, 313, 810

Kulkarni, S. R. 1988, Adv. Space Res., 8, 343

Kulkarni, S. R., \& van Kerkwijk, M. H. 1998, ApJ, 507, L49

Marsh, M. C., et al. 1997, MNRAS, 286, 369

Melatos, A. 1997, MNRAS, 288, 1049

Mignani, R. P. 1998, in Neutron Stars and Pulsars: Thirty Years after the Discovery, ed. N. Shibazaki, N. Kawai, S. Shibata, \& T. Kifune (Tokyo: Universal Academic Press), 335

Monet, D., et al. 1998, USNO-A2.0 (Washington DC: US Naval Observatory)

Oke, J. B., et al. 1995, PASP, 107, 375
Phinney, E. S., \& Verbunt, F. 1991, MNRAS, 248, 21P

Piran, T. 1997, in Unsolved Problems in Astrophysics, ed. J. Bahcall \& J. P. Ostriker (Princeton: Princeton Univ. Press), 343

Provencal, J. L., Shipman, H. L., Hog, E., \& Thejll, P. 1998, ApJ, 494, 759 Schaller, G., Schaerer, D., Meynet, G., \& Maeder, A. 1992, A\&AS, 96, 269 Schlegel, D. J., Finkbeiner, D. P., \& Davis, M. 1998, ApJ, 500, 525

Schmidt, G. D., Bergeron, P., Liebert, J., \& Saffer, R. A. 1992, ApJ, 394, 603

Starrfield, S. 1989, in Classical Novae, ed. N. Evans \& M. Bode (New York: Wiley), 123

Stokes, G. H., Taylor, J. H., \& Dewey, R. J. 1985, ApJ, 294, L21

Taylor, J. H., \& Cordes, J. M. 1993, ApJ, 411, 674

Taylor, J. H., Wolszczan, A., Damour, T., \& Weisberg, J. M. 1992, Nature, 355,132

Thorsett, S. E., Arzoumanian, Z., McKinnon, M. M., \& Taylor, J. H. 1993, ApJ, 405, L29

Thorsett, S. E., \& Chakrabarty, D. 1999, ApJ, 512, 288

van den Heuvel, E. P. J. 1995, J. Astrophys. Astron., 16, 255

Vennes, S., Bowyer, S., \& Dupuis, J. 1996, ApJ, 461, L103

Vennes, S., Thejll, P., Genova-Galvan, R., \& Dupuis, J. 1997, ApJ, 480, 714

Wood, M. A. 1995, in Proc. 9th European Workshop on White Dwarfs, ed. D. Koester \& K. Werner (Berlin: Springer), 41 